\title{
How extensive of a 4D dataset is needed to estimate cumulative dose distribution plan evaluation metrics in conformal lung therapy? ${ }^{\text {a) }}$
}

Mihaela Rosu, ${ }^{\text {b) }}$ James M. Balter, Indrin J. Chetty, ${ }^{c)}$ Marc L. Kessler, and Daniel L. McShan

The University of Michigan, Department of Radiation Oncology, Ann Arbor, Michigan 48109-0010

Peter Balter

The University of Texas M.D. Anderson Cancer Center, Department of Radiation Physics, Houston, Texas 77030-0547

Randall K. Ten Haken

The University of Michigan, Department of Radiation Oncology, Ann Arbor, Michigan 48109-0010

(Received 1 March 2006; revised 24 October 2006; accepted for publication 24 October 2006; published 20 December 2006)

The purpose of this study was to investigate the number of intermediate states required to adequately approximate the clinically relevant cumulative dose to deforming/moving thoracic anatomy in four-dimensional (4D) conformal radiotherapy that uses $6 \mathrm{MV}$ photons to target tumors. Four patients were involved in this study. For the first three patients, computed tomography images acquired at exhale and inhale were available; they were registered using B-spline deformation model and the computed transformation was further used to simulate intermediate states between exhale and inhale. For the fourth patient, 4D-acquired, phase-sorted datasets were available and each dataset was registered with the exhale dataset. The exhale-inhale transformation was also used to simulate intermediate states in order to compare the cumulative doses computed using the actual and the simulated datasets. Doses to each state were calculated using the Dose Planning Method (DPM) Monte Carlo code and dose was accumulated for scoring on the exhale anatomy via the transformation matrices for each state and time weighting factors. Cumulative doses were estimated using increasing numbers of intermediate states and compared to simpler scenarios such as a "2-state" model which used only the exhale and inhale datasets or the dose received during the average phase of the breathing cycle. Dose distributions for each modeled state as well as the cumulative doses were assessed using dose volume histograms and several treatment evaluation metrics such as mean lung dose, normal tissue complication probability, and generalized uniform dose. Although significant "point dose" differences can exist between each breathing state, the differences decrease when cumulative doses are considered, and can become less significant yet in terms of evaluation metrics depending upon the clinical end point. This study suggests that for certain "clinical" end points of importance for lung cancer, satisfactory predictions of accumulated total dose to be received by the distorting anatomy can be achieved by calculating the dose to but a few (or even simply the average) phases of the breathing cycle. (c) 2007 American Association of Physicists in Medicine. [DOI: 10.1118/1.2400624]

Key words: organ deformation, 4D treatment planning, 4D datasets, Monte Carlo

\section{INTRODUCTION}

Breathing motion and organ deformation may lead to geometric misses that have the potential of underdosing tumor and overdosing healthy tissue, when conformal radiation therapy is used to treat tumors located in thorax. Historically, the three-dimensional (3D) anatomical description of the patient used for treatment planning was acquired during free breathing computed tomography (CT) scanning, and thus was affected by motion artifacts. ${ }^{1-3}$ This, in turn, made target definition prone to errors and inaccuracies, as was the estimation of the dose to be received by the patient. The next natural step was to add a temporal component to the imaging process. Simple voluntary breath hold techniques or more sophisticated ones such as active breathing control $^{3,4}$ and deep inspiration breath hold, ${ }^{5-7}$ improved the quality of the CT images, but usually limited the geometrical information to just one phase of the breathing cycle. As a result, the subsequent delivery of the radiation only during a selected phase or portion of the breathing cycle significantly reduced the duty cycle.

In the past few years, the development of the fourdimensional (4D) CT scanning technology made possible the generation of sequential image datasets for multiple phases of the breathing cycle. ${ }^{8-10}$ Integration of similar concepts and tools into radiation therapy has led to $4 \mathrm{D}$ radiotherapy, defined, for example, ${ }^{11}$ as the "explicit inclusion of the temporal changes of anatomy during the imaging, planning and delivery of radiotherapy." Within such a regimen, tumor motion could be accommodated, for example, by tracking tumors in real time and by adjusting the beam delivery accordingly based on plans individually designed for each available instance of the patient anatomy encountered during a respi- 
ratory cycle. Unfortunately, the practicality of this adaptive approach may be limited due to difficulties associated with the prediction of the future target positions and the repositioning of the beam in a timely manner. ${ }^{12,13}$ On the other hand, even if all the technical considerations were dealt with, such techniques would still require patients that are capable of complying and are involved actively in the treatment process; a situation that may not be the case, especially in lung cancer treatment. Therefore, treating a freely breathing patient nonadaptively in the real-time sense aforementioned is still an appealing option, the radiation therapy in this case being truly $4 \mathrm{D}$ only at the time of imaging.

Even a simplified delivery process would still benefit from the time dependent information acquired during imaging in order to better predict the doses to be received by a deforming anatomy in the presence of the intra-fraction respiratory motion. For these studies, out of all datasets available after simulation, one is chosen as the planning ("static") dataset and is used to design a treatment plan. This plan (i.e., beam arrangement and intensities) is then used for dose calculations on the other datasets and finally, the doses from all datasets are scored on the planning dataset, with the appropriate time weighting coefficients.

The purpose of this study was to investigate how many (or rather few) 4D scan phases are required in order to adequately estimate, in the planning process, the clinically relevant cumulative dose to be received by a deforming/moving thoracic anatomy during free breathing radiation delivery. The cumulative doses, estimated in several scenarios, using a decreasing number of datasets, were compared through their impact on treatment evaluation metrics widely employed in lung cancer treatment planning, such as the tumor equivalent uniform dose, mean lung dose, and normal lung tissue complication probability.

The accuracy of the cumulative dose depends on the accuracy of the registration process between various scans available. Therefore, in order to avoid bias due to even small registration errors, the use of simulated datasets between exhale and inhale was preferred. In addition to that, one case was analyzed using actual phase-sorted 4D data, as well as simulated data. Here the intents were also: (a) to compare the cumulative dose based on actual and simulated data; (b) to investigate if the cumulative dose in the presence of breathing-induced hysteresis could be predicted with a sufficient degree of accuracy by assuming a linear trajectory between exhale and inhale.

\section{METHODS AND MATERIALS}

\section{A. Patient data}

For three patients-referred to as Patients A, B, and C throughout the paper-the treatment planning data used in this study were obtained from patients diagnosed with inoperable nonsmall cell lung cancer, under a protocol approved by the Internal Review Board at the University of Michigan. Input data consisted of CT scans acquired sequentially in the same session at normal exhale and inhale states during coached voluntary breath hold. No breathing pattern infor- mation was available for these patients, and therefore we have assumed the analytical breathing function: ${ }^{14} z(t)=z_{0}$ $-b \cos ^{2 n}\left(\frac{\pi t}{\tau}\right)$, with $z_{0}$ the exhale coordinate, $b$ the breathing amplitude, $\tau$ the breathing period and $n$ a fitting parameter describing the asymmetry of the respiratory pattern. The subsequent analyses were performed for both $n=1$ and $n=3$, describing symmetrical and asymmetrical (biased toward exhale) breathing cycles, respectively.

For the fourth patient, referred to as Patient D, a 4D dataset was acquired using a multislice CT scanner (Discovery ST, General Electric Healthcare systems, Wausheka, WI), as part of a routine protocol for lung cancer patients, in place at University of Texas M.D. Anderson Cancer Center. The 4D data were sorted in ten datasets between exhale and inhale, using the phase of respiratory cycle as recorded by a real-time positioning system (Varian Medical Systems, Palo Alto, CA). ${ }^{8}$ Phases were assigned as illustrated in Fig. 1, such that each dataset was comprised of images acquired at and around a given time during a breathing period. These phase-sorted datasets were assumed to be free of artifacts.

The patients were selected such that they had different, representative tumor locations and larger motion amplitudes, as indicated in Table I. The breathing-induced tumor motion amplitudes were determined based on the displacement of the center of the mass of the tumor between the exhale and inhale datasets.

\section{B. Image registration}

For this study, the exhale dataset was chosen to be the reference dataset. As such, for dose accumulation, all other datasets were registered with the exhale dataset using a B-spline technique. ${ }^{15-17}$ This methodology is believed to be appropriate for describing nonrigid changes due to its ability to deal with local deformations, such as those encountered in lungs as a result of respiration. The registration process started by placing a regular coarse grid of control points (knots) across the thorax in both datasets and then a gradient descent algorithm ${ }^{18}$ was used to vary the cubic B-spline's coefficients until the registration metric (average sum of squared differences of the intensities between two dataset images) was minimized. After initial convergence, the knot grid spacing was subdivided by two and the process continued and then reiterated until the knot spacing fell below a predefined threshold. ${ }^{16,17}$ The registration process is "local," in the sense that perturbing the position of any point only affects the transformation in the neighborhood of that point; a direct consequence of the limited domain over which the basis functions of a B-spline transformation are defined. ${ }^{16}$

\section{Intermediate states data simulation}

For Patients A, B, and C, datasets for intermediate states between inhale $(0 \%)$ and exhale $(50 \%)$ were simulatedfrom $5 \%$ to $45 \%$ in excursion away from the inhale state, in $5 \%$ increments - by using the transformation provided by the registration between the real exhale and inhale datasets and assuming linear displacements along that path (see Fig. 1). The data simulated by assuming linear displacements mimic 


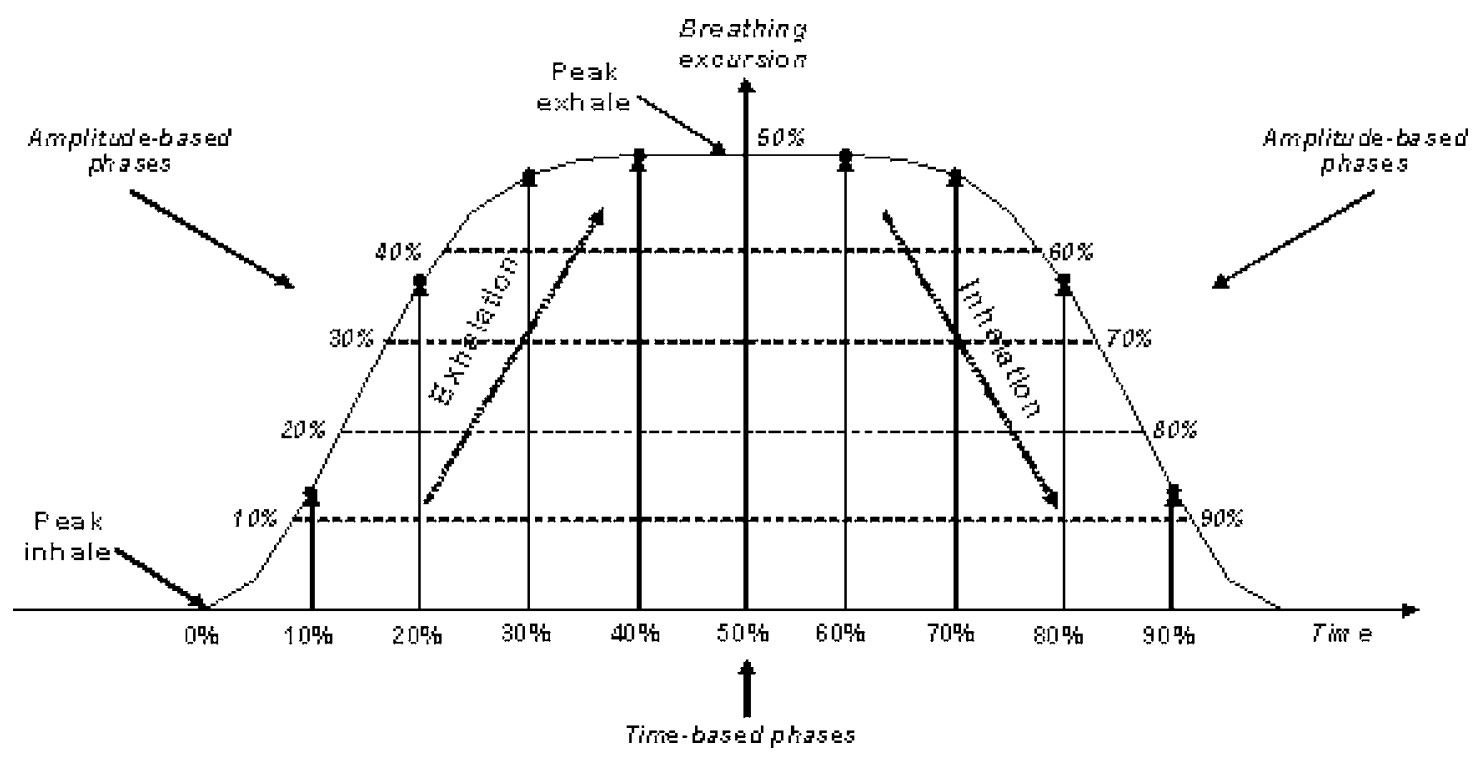

FIG. 1. Phase labeling in 4D: (a) by time- breathing period is divided into equal intervals and a phase that represents a percentage of the breathing period is assigned to each time interval; (b) by amplitude-breathing excursion is divided into equal intervals and a phase that represents a percentage of the breathing amplitude is assigned to each amplitude interval.

actual data that would be sorted by amplitude. ${ }^{19,20}$ By assuming linear displacements between exhale and inhale we implicitly assumed no hysteresis, that is, no difference between the exhalation and inhalation tumor trajectory. Therefore, the $5 \%-45 \%$ states from the inhalation segment of the breathing cycle also represent the $95 \%-55 \%$ states from the exhalation. The feasibility of using B-spline nonrigid registration to generate the intermediate phases by interpolating between two sets of 3D CT images acquired at different phases has been also reported recently by Schreibmann, Chen, and Xing. ${ }^{21}$ In order to avoid bias due to any exhale/inhale registration errors and to assess the quality of the initial exhale-inhale distorted image transformation fit, we also simulated the inhale dataset and compared it to the real, imaged inhale dataset. For dose calculation the lungs were contoured on all simulated images and the mean relative electron densities were scaled accordingly, such that the mass of the each lung was conserved.

The same procedure was used to generate intermediate states for Patient D as well.

\section{Monte Carlo treatment planning}

The treatment plans were designed on the exhale dataset (planning dataset) and consisted of $6 \mathrm{MV}$ anterior, lateral and oblique fields, combined with segmental fields directed from the same angles to produce a dose distribution within the planning target volume (PTV) of $100 \% \pm 5 \%$ of the prescription dose. For this study, the PTVs were generated by uniformly expanding the target by $1 \mathrm{~cm}$. The number of beam segments per plan was five for Patient A, seven for Patients B and C, and eight for Patient D, with no preferential relative orientation of the beams with respect to the tumor direction of motion. Dose distributions were calculated using the Dose Planning Method (DPM) Monte Carlo code, ${ }^{22,23}$ available within our in-house treatment planning system (UMPlan). We used a $2 \mathrm{~mm}$ step size, and low energy electron and photon cutoff values of 200 and $50 \mathrm{keV}$, respectively. For each treatment plan, 1.5 billion histories per plan were simulated, resulting in $1-\sigma$ statistics of better than $0.5 \%$ on average within the PTV. ${ }^{24}$ All plans were normalized to $100 \%$ at the isocenter. These conformal plans (beam

TABLE I. Patient information regarding tumor position and motion amplitude.

\begin{tabular}{lccc}
\hline \hline Patient & Tumor location & $\begin{array}{c}\text { Dominant direction } \\
\text { of motion with } \\
\text { respect to exhale }\end{array}$ & Motion amplitude \\
\hline A & Apical & Superior & $1.0 \mathrm{~cm}$ \\
B & Medial, embedded in normal lung & Anterior & $0.7 \mathrm{~cm}$ \\
C & Near diaphragm & Inferior & $2.0 \mathrm{~cm}$ \\
D & Medial & Inferior & $0.7 \mathrm{~cm}$ \\
\hline \hline
\end{tabular}


TABLE II. Time-weighting coefficients for the simulated and actual data. For the simulated data, the coefficients are derived from the breathing probability distribution function for $n=1$ and $n=3$. For the actual data, all phases are equally weighted. The subscripts indicate the breathing phase.

\begin{tabular}{|c|c|c|c|c|c|c|c|c|}
\hline & \multicolumn{6}{|c|}{ Simulated data } & \multicolumn{2}{|c|}{ Actual data } \\
\hline & \multicolumn{3}{|c|}{$n=1$} & \multicolumn{3}{|c|}{$n=3$} & \multirow[b]{2}{*}{$\begin{array}{c}\text { "2- } \\
\text { states" } \\
\text { model }\end{array}$} & \multirow[b]{2}{*}{$\begin{array}{l}\text { "10- } \\
\text { states" } \\
\text { model }\end{array}$} \\
\hline & $\begin{array}{c}\text { "2- } \\
\text { states" } \\
\text { model }\end{array}$ & $\begin{array}{c}\text { "10- } \\
\text { states" } \\
\text { model }\end{array}$ & $\begin{array}{l}\text { "20- } \\
\text { states" } \\
\text { model }\end{array}$ & $\begin{array}{c}\text { "2- } \\
\text { states" } \\
\text { model }\end{array}$ & $\begin{array}{c}\text { "10- } \\
\text { states" } \\
\text { model }\end{array}$ & $\begin{array}{c}\text { “20- } \\
\text { states" } \\
\text { model }\end{array}$ & & \\
\hline$w_{0}$ & 0.5 & 0.205 & 0.144 & 0.3 & 0.119 & 0.083 & 0.5 & 0.1 \\
\hline$w_{5}$ & & & 0.055 & & & 0.032 & & \\
\hline$w_{10}$ & & 0.082 & 0.04 & & 0.049 & 0.024 & & 0.1 \\
\hline$w_{15}$ & & & 0.035 & & & 0.0215 & & \\
\hline$w_{20}$ & & 0.0665 & 0.0325 & & 0.0415 & 0.0205 & & 0.1 \\
\hline$w_{25}$ & & & 0.032 & & & 0.021 & & \\
\hline$w_{30}$ & & 0.0665 & 0.0325 & & 0.045 & 0.0225 & & 0.1 \\
\hline$w_{35}$ & & & 0.035 & & & 0.0255 & & \\
\hline$w_{40}$ & & 0.082 & 0.04 & & 0.0665 & 0.032 & & 0.1 \\
\hline$w_{45}$ & & & 0.055 & & & 0.0525 & & \\
\hline$w_{50}$ & 0.5 & 0.205 & 0.144 & 0.7 & 0.477 & 0.415 & 0.5 & 0.1 \\
\hline$w_{55}$ & & & 0.055 & & & 0.0525 & & \\
\hline$w_{60}$ & & 0.082 & 0.04 & & 0.0665 & 0.032 & & 0.1 \\
\hline$w_{65}$ & & & 0.035 & & & 0.0255 & & \\
\hline$w_{70}$ & & 0.0665 & 0.0325 & & 0.045 & 0.0225 & & 0.1 \\
\hline$w_{75}$ & & & 0.032 & & & 0.021 & & \\
\hline$w_{80}$ & & 0.0665 & 0.0325 & & 0.0415 & 0.0205 & & 0.1 \\
\hline$w_{85}$ & & & 0.035 & & & 0.0215 & & \\
\hline$w_{90}$ & & 0.082 & 0.04 & & 0.049 & 0.024 & & 0.1 \\
\hline$w_{95}$ & & & 0.055 & & & 0.032 & & \\
\hline
\end{tabular}

directions, weights, apertures and number of monitor units) designed on the planning dataset were also used for the calculation of the dose distribution on all simulated datasets at the same dose grid resolution. The dose grid size in all dimensions was $3.5 \mathrm{~mm}^{25}$

\section{E. Dose accumulation}

The accumulation of doses from the intermediate datasets to the exhale planning dataset was performed as described elsewhere. $^{25}$ In brief, the volume associated with each exhale voxel is first subdivided into octants, the center of each octant is mapped to locations on the intermediate state dose grid, doses at the tracked locations are estimated by trilinear interpolation and their average values are scored at the original exhale dose grid point locations.

In cases where simulated data were used, the doses from intermediate states were mapped back onto the exhale dataset based on the exhale-inhale transformation, assuming that for each simulated state the displacement was the corresponding fraction of the exhale-inhale excursion, much like the approach used to simulate the data. In the case of Patient D, when the actual 4D data were used, the dose from each phase was mapped onto the exhale dataset using the transformation provided by the registration between each intermediate dataset and exhale.

The accumulation of doses in the deforming anatomy was performed by applying time weighting factors: $D_{\text {rec }}(i)$ $=\Sigma_{k} w_{k} \cdot D_{k}(i)$, where $D_{\text {rec }}(i)$ is the cumulative dose in the exhale voxel $i, k$ the breathing phase, $D_{k}(i)$ the dose received by deformed exhale voxel $i$ at the breathing phase $k, w_{k}$ the time weighting coefficient at breathing stage $k$. The corresponding time weighting coefficients used in the dose reconstruction are presented in Table II. For the simulated data, the coefficients were derived from the breathing probability distribution function ${ }^{14}$ for $n=1$ and $n=3$. For the actual data, all phases are equally weighted in time, since that is the very nature of the phase-based data sorting.

The cumulative doses, reported on the exhale planning CT, were estimated in several scenarios using different numbers of intermediate states, with corresponding adjustment of the associated time weighting coefficients used in the dose reconstruction:

(1) by considering only the exhale and inhale geometries ("2-state dose");

(2) by considering only the geometries at the time-weighted average breathing phases from the first and the second halves of the time interval between exhale and inhale ("2-ave state dose"). Based on the abovementioned breathing function ${ }^{14}$ these average positions, during the inhalation segment, were found to be $\sim 10 \%$ and $\sim 45 \%$ from the inhale position for $n=3$, and $\sim 10 \%$ and $\sim 40 \%$ for $n=1$, and their symmetrical values with respect to the $50 \%$ state during exhalation;

(3) by including exhale, inhale and the 10\%-90\% geometries ("10-state dose"); 
TABLE III. Scenarios used to estimate cumulative doses. The crosses indicate states that were used in any given scenario. For the actual 4D data (Patient D), the phase indicates time equi-spaced datasets over a breathing period. For the simulated data (Patients A-D), the phase indicates spatially evenly distributed datasets over the breathing amplitude. $n=1$ and $n=3$ describe symmetrical and asymmetrical (biased toward exhale) breathing cycles, respectively.

\begin{tabular}{|c|c|c|c|c|c|c|c|c|c|c|c|}
\hline \multirow[b]{4}{*}{ Phase } & \multicolumn{7}{|c|}{ Patients A, B, C } & \multicolumn{4}{|c|}{ Patient D } \\
\hline & \multirow[b]{3}{*}{$\begin{array}{c}\text { "20 } \\
\text { states" }\end{array}$} & \multirow[b]{3}{*}{$\begin{array}{c}\text { "10 } \\
\text { states" }\end{array}$} & \multirow[b]{3}{*}{$\begin{array}{c}\text { "2 } \\
\text { states" }\end{array}$} & \multicolumn{4}{|c|}{ Scenarios } & & & & \\
\hline & & & & \multicolumn{2}{|c|}{$\begin{array}{l}\text { "2-ave } \\
\text { states" }\end{array}$} & \multicolumn{2}{|c|}{$\begin{array}{l}\text { "Ave } \\
\text { state" }\end{array}$} & \multicolumn{2}{|c|}{ Actual data } & \multicolumn{2}{|c|}{ Simulated data } \\
\hline & & & & $n=1$ & $n=3$ & $n=1$ & $n=3$ & $\begin{array}{c}\text { "10 } \\
\text { states" }\end{array}$ & $\begin{array}{c}" 2 \\
\text { states" }\end{array}$ & $\begin{array}{c}\text { "10 } \\
\text { states" }\end{array}$ & $\begin{array}{c}\text { "2 } \\
\text { states" }\end{array}$ \\
\hline $0 \%$ (Inhale) & $\mathrm{x}$ & $\mathrm{x}$ & $\mathrm{x}$ & & & & & $\mathrm{x}$ & $\mathrm{x}$ & $\mathrm{x}$ & $\mathrm{x}$ \\
\hline $5 \%$ & $\mathrm{x}$ & & & & & & & & & & \\
\hline $10 \%$ & $\mathrm{x}$ & $\mathrm{x}$ & & $\mathrm{x}$ & $\mathrm{x}$ & & & $\mathrm{x}$ & & $\mathrm{x}$ & \\
\hline $15 \%$ & $\mathrm{x}$ & & & & & & & & & & \\
\hline $20 \%$ & $\mathrm{x}$ & $\mathrm{x}$ & & & & & & $\mathrm{x}$ & & $\mathrm{x}$ & \\
\hline $25 \%$ & $\mathrm{x}$ & & & & & & $\mathrm{x}$ & & & & \\
\hline $30 \%$ & $\mathrm{x}$ & $\mathrm{x}$ & & & & & & $\mathrm{x}$ & & $\mathrm{x}$ & \\
\hline $35 \%$ & $\mathrm{x}$ & & & & & $\mathrm{x}$ & & & & & \\
\hline $40 \%$ & $\mathrm{x}$ & $\mathrm{x}$ & & $\mathrm{x}$ & & & & $\mathrm{x}$ & & $\mathrm{x}$ & \\
\hline $45 \%$ & $\mathrm{x}$ & & & & $\mathrm{x}$ & & & & & & \\
\hline $50 \%$ (Exhale) & $\mathrm{x}$ & $\mathrm{x}$ & $\mathrm{x}$ & & & & & $\mathrm{x}$ & $\mathrm{x}$ & $\mathrm{x}$ & $\mathrm{x}$ \\
\hline $55 \%$ & $\mathrm{x}$ & & & & $\mathrm{x}$ & & & & & & \\
\hline $60 \%$ & $\mathrm{x}$ & $\mathrm{x}$ & & $\mathrm{x}$ & & & & $\mathrm{x}$ & & $\mathrm{x}$ & \\
\hline $65 \%$ & $\mathrm{x}$ & & & & & $\mathrm{x}$ & & & & & \\
\hline $70 \%$ & $\mathrm{x}$ & $\mathrm{x}$ & & & & & & $\mathrm{x}$ & & $\mathrm{x}$ & \\
\hline $75 \%$ & $\mathrm{x}$ & & & & & & $\mathrm{x}$ & & & & \\
\hline $80 \%$ & $\mathrm{x}$ & $\mathrm{x}$ & & & & & & $\mathrm{x}$ & & $\mathrm{x}$ & \\
\hline $85 \%$ & $\mathrm{x}$ & & & & & & & & & & \\
\hline $90 \%$ & $\mathrm{x}$ & $\mathrm{x}$ & & $\mathrm{x}$ & $\mathrm{x}$ & & & $\mathrm{x}$ & & $\mathrm{x}$ & \\
\hline $95 \%$ & $\mathrm{x}$ & & & & & & & & & & \\
\hline
\end{tabular}

(4) by including exhale, inhale and the 5\%-95\% geometries ("20-state dose");

(5) by using only the geometry associated with the timeweighted average phase over the complete breathing cycle ("ave-state" dose). For the breathing model assumed in this study these average states were found to be (for the inhalation) at $\sim 35 \%$ from the inhale position for $n=3$ and at $25 \%$ for $n=1$, and their symmetrical values with respect to the $50 \%$ state during exhalation.

All scenarios are summarized in Table III, where the crosses indicate states that were used in any given scenario. For the actual 4D data (Patient D), the phase indicates time equi-spaced datasets over the breathing period. For the simulated data (Patients A-D), the phase indicates spatially evenly distributed datasets over the breathing amplitude.

The cumulative doses compiled in all scenarios were compared through dose difference displays in order to estimate point dose differences, through dose volume histograms (DVHs) for the structure of interest and through clinical outcome metrics such as generalized uniform $\operatorname{dose}^{26}$ (gEUD) for the targets, mean lung dose (MLD) and normal tissue complication probabilities (NTCPs). The tumor gEUDs were evaluated using an " $a$ " parameter value of -10 , which assumes a moderately aggressive tumor. NTCP was calculated using the Lyman model ${ }^{27}$ based on the effective volume
DVH reduction methodology described by Kutcher and Burman. ${ }^{28}$ The NTCP model parameters for the normal lung: $T D(50)=30.8 \mathrm{~Gy}$ at $2.0 \mathrm{~Gy}$ per fraction, $m=0.99$, and $n$ $=0.37$, were acquired from a previous study. ${ }^{29}$

\section{RESULTS}

\section{A. Registration assessment}

The exhale-inhale B-spline registration results, as assessed by visual inspection, were found to be satisfactory for all patients. The example shown in Fig. 2 illustrates for Patient $\mathrm{C}$, who underwent the largest tumor motion excursion, the results of an inhale coronal image aligned without deformation to the corresponding exhale reference image, and then registered with deformation. The appropriateness of using the simulated images was also assessed by comparing the tumor and normal lung DVHs for dose distributions computed on both the real and simulated inhale datasets, for which both types of data were available. The cumulative DVHs were found (data not shown here) to be nearly identical for each of the patients.

For Patient D, the top row in Fig. 3 displays coronal cuts generated from the 4D datasets through the same plane in the room coordinate reference system. The bottom row in the same figure displays the coronal cuts generated from the 

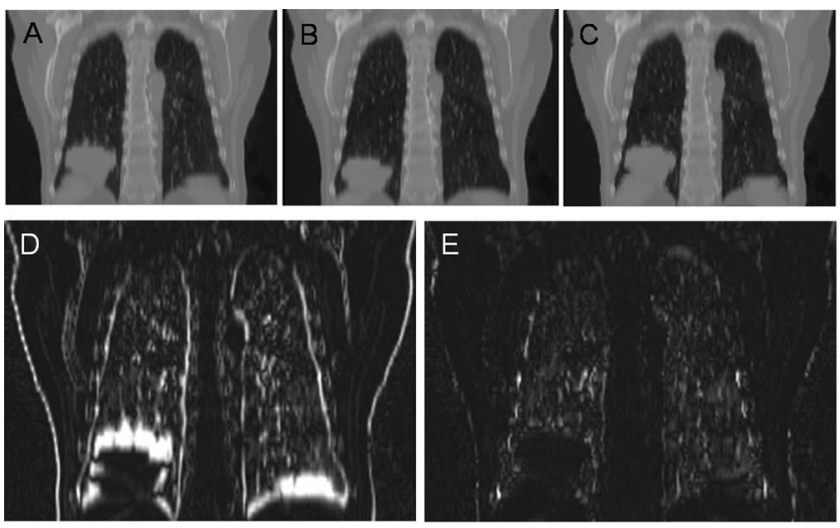

FIG. 2. B-spline registration results for Patient $\mathrm{C}$ shown for an example coronal cut: (A) exhale image; (B) inhale image; (C) inhale distorted to form the corresponding exhale image; (D) exhale and inhale shown together before registration; (E) exhale and distorted inhale shown together after registration. The lighter regions in $\mathrm{D}$ and $\mathrm{E}$ correspond to registration mismatches between exhale and distorted inhale.

simulated datasets. Although the actual 4D data represent phase-sorted images, whereas the simulated data correspond to amplitude binned data, the similarity and consistency of the simulated images and the actual images is obvious. The displacement of the top of the tumor was less evident in the simulated datasets, due to an imperfect registration in that region, attributed to the more diffuse tumor extent; the misalignment in that region, although not considered severe, was identified visually when the exhale and the deformed inhale datasets for this patient were displayed together after registration.

\section{B. Comparison of cumulative doses in various scenarios}

As stated in the Methods section, the distorted B-spline transformation resulting from the exhale-inhale registration was subsequently used to simulate distorted geometries for various other, intermediate phases of the breathing cycle on which the dose distribution was recomputed. The transformations were then used together with the time-weighting factors to compile the cumulative dose received over the breathing cycle (computed on each simulated "nonexhale" dataset) back onto the original exhale dataset. As expected (e.g., for Patient A in Fig. 4), dose difference maps between each state simulated during inhalation scored back onto the exhale scan, and the original planning exhale dose exhibit increasing magnitudes of change from the original exhale dose calculation as the simulated states get further away from the exhale position (get nearer to inhale). These changes range from a few percent for CT45 (the 5\% geometric change from exhale state) to $\sim 40 \%$ for the inhale state.

Figure 5 shows, for Patient $\mathrm{C}$ and for $n=3$, dose difference displays between the "20-state" cumulative dose, that is, the most accurate reproduction of the cumulative dose in our investigation, and the other cumulative dose distributions- " 10 state," the " 2 state," the "2-ave state," and the "ave state" (CT35 in this case) doses-all scored back onto the original exhale state. The differences between the inhale state and the exhale state, and the " 20 -state" cumulative dose and the exhale planned state are also displayed to illustrate the magnitude of the observed differences. The DVHs for the intermediate state dose distributions mapped back onto the exhale state, shown in Fig. 6, spread between the exhale and transformed inhale DVHs. However, as it can be seen in the insets, the DVHs for the cumulative dose distributions are close together, with no clear separation, irrespective of the method used for the prediction of the cumulative dose.

The trends described above were observed for Patients A, B, and C for both $n=1$ and $n=3$. A summary of tumor EUDs and mean lung doses (for all patients) and esophagus NTCPs (only an issue for patient A) for all dose accumulation scenarios are presented in Table IV.

For Patient D, displayed in Fig. 7 are dose differences between doses computed on each dataset from the 4D scan (mapped back onto the exhale dataset) and the exhale dose, as well as dose differences between doses calculated on the simulated intermediate states (mapped also back onto the exhale dataset) and the exhale dose. In the latter case, only differences corresponding to datasets simulated for the inhalation part of the respiratory cycle are shown, as the simulated datasets are identical for both inhalation and exhalation. For the actual 4D data analysis, it can be noticed that, while the changes in dose from the exhale values during inhalation/

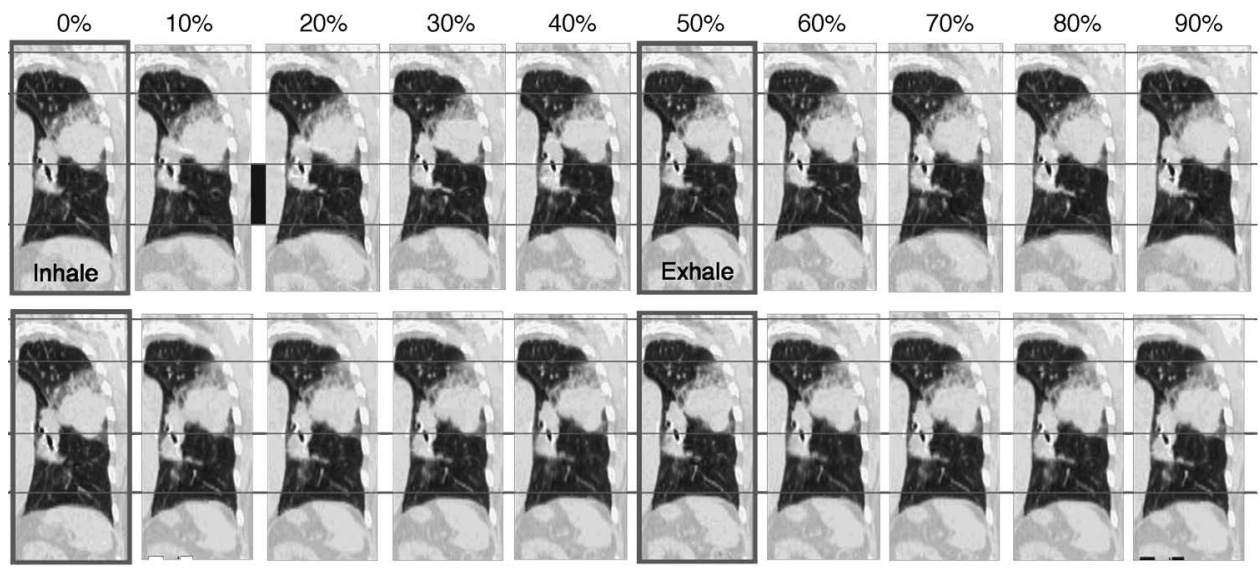

FIG. 3. Patient D. Example of coronal cuts generated from the actual 4D datasets (top row) and from the simulated datasets (bottom row) through the same plane in the room coordinate reference system. For the actual dataset, the numbers represent timebased phase over a breathing period, while for the simulated datasets, the numbers indicate amplitude-based phase, as indicated in Fig. 1. 

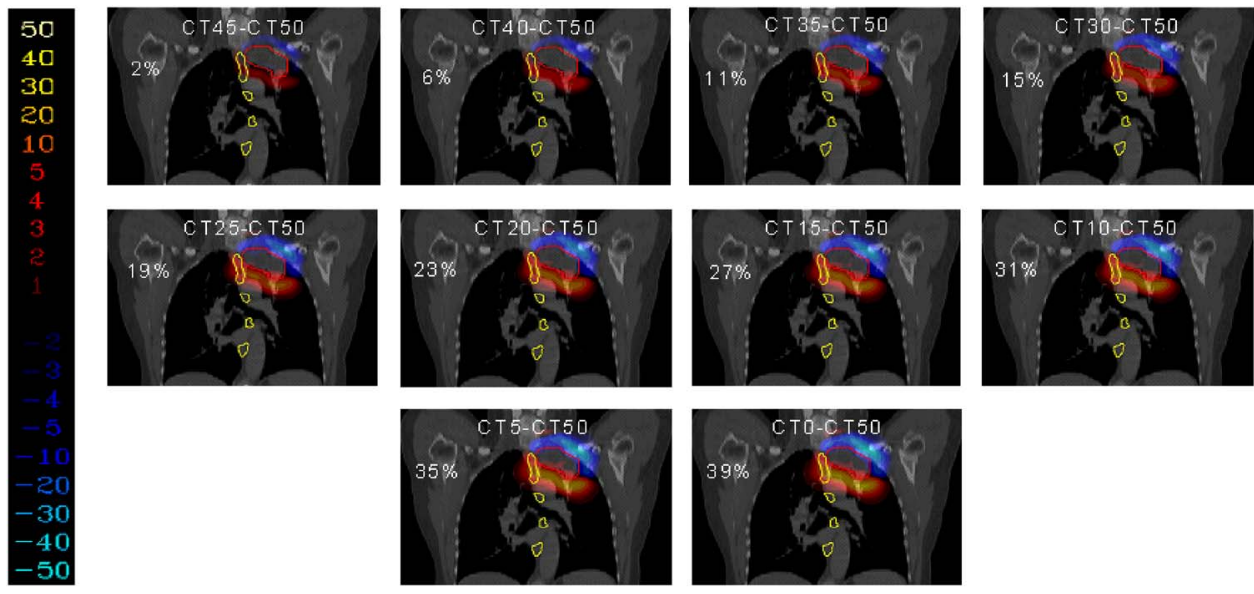

FIG. 4. Patient A: difference maps between doses in each simulated state during inhalation (scored at exhale) and the planning exhale dose. Inhale and exhale are the $0 \%$ and $50 \%$ phases respectively. The numbers indicate maximum point dose differences inside the lung. Red/blue spots indicate positive/negative differences. exhalation are consistent with the magnitude of the anatomical changes undergone during respiration, the location of the hot and cold spots that characterize the motion/deformation effects is somewhat different during inhalation as compared to exhalation-a dosimetric illustration of the respiratory hysteresis effect.

Shown in Fig. 8 are dose differences between cumulative doses estimated in two scenarios ("10 state" and "2 state") and the static exhale dose, for both the actual 4D data and the simulated data, again for Patient D. In all cases, similar differences - with respect to their magnitude and locationbetween cumulative doses and the static exhale dose were found. The differences between the "10 state" and "2 state" doses (not shown here) were less than $2 \%$ between any scenario.

The clinical target volume (CTV) and normal lung DHVs for Patient D are illustrated in Fig. 9 for the intermediate state doses scored onto the exhale dataset and cumulative doses, for the actual data and for the simulated data. Just as for Patients A-C, the cumulative dose DVHs are similar to each other, regardless of the number of intermediate states used for dose reconstruction. The treatment metrics evaluated for all these dose distributions (gEUD, MLD, NTCP) are reported on the bar plots shown in Fig. 10. Again, the treatment metrics are almost identical for the "10 state" and "2 state" scenarios. Moreover, the metrics associated with the actual dataset compare favorably to those derived for the simulated datasets.

\section{DISCUSSION}

The goal of this study was to investigate the number of respiratory phases needed to estimate, before the onset of the treatment, the clinically relevant cumulative doses to thorax that would be received by a patient if the treatment was delivered during free breathing. The patients involved in the study were selected such that they were representative in terms of tumor location and motion amplitude. The use of simulated data to describe intermediate breathing phases was preferred in order to eliminate any bias in the cumulative dose due to registration errors, given that to date there is no complete, rigorous way to fully assess the registration accuracy. The images derived by interpolation, however, offered a good description of the respiratory induced anatomical changes, as it was seen for Patient D. As a figure of merit, the simulated data provided a finer sampling of the various ge-

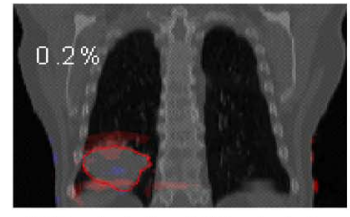

"20-state" - "10-state"

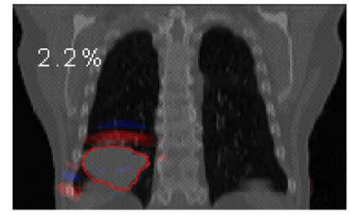

"20-state" - "2-state"

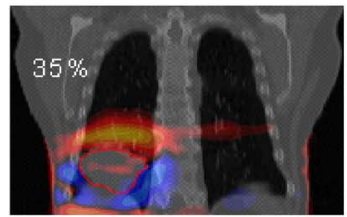

Inhale $(0 \%)$ - Exhale $(50 \%)$

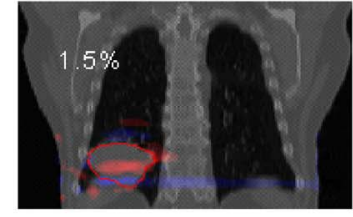

"20-state" - "2-ave state"

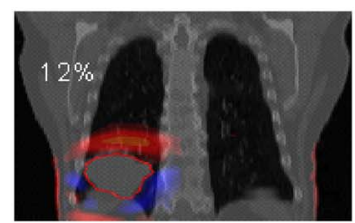

"20-state"- Exhale (50\%)

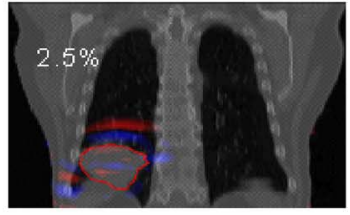

"20-state" - "ave state"

FIG. 5. Patient C, $n=3$ : dose difference maps between: cumulative doses estimated in various scenarios; inhale and exhale doses; cumulative and exhale doses. The numbers indicate maximum point dose differences inside the lung. Red/blue spots indicate positive/negative differences (same color code as shown in Fig. 4). 


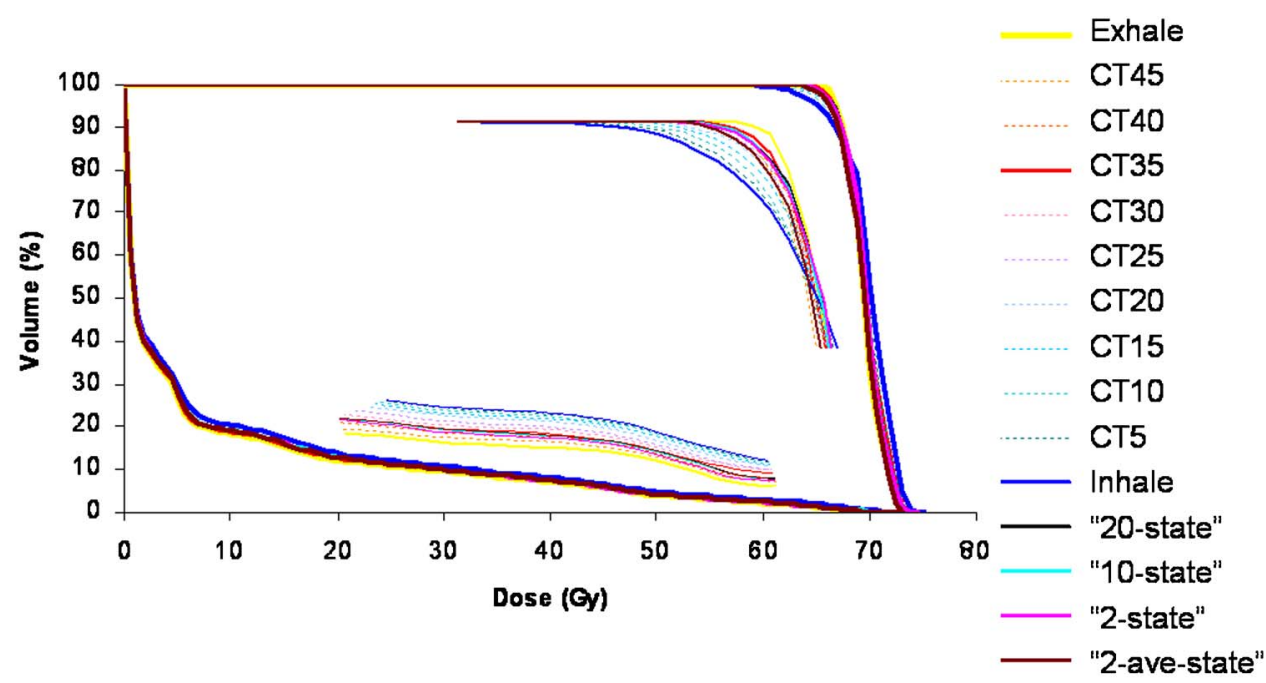

FIG. 6. Patient C, $n=3$ : cumulative DVHs for tumor and normal lung tissue with insets showing details.

ometries between exhale and inhale, as opposed to the actual 4D phase-sorted images. By following the most inferior guideline in Fig. 3, it can be noticed that the datasets sorted from the actual 4D data comprised several exhale-like datasets (i.e., 30\%, 40\%, 50\%, 60\%) and several inhale-like datasets (i.e., $0 \%, 10 \%, 90 \%$ ), whereas the simulated data captured better the gradual progression of the diaphragm from the exhale to the inhale configuration. This is not unexpected: a phase-based data sorting procedure will sample more states in the vicinity of exhale and inhale as more time is usually spent at and around these phases during the respiratory cycle, as illustrated in Fig. 1 as well. Similar conclusions regarding the goodness of modeling intermediate states through interpolation has also been pointed out by Schreibmann, Chen, and Xing. ${ }^{21}$

The major drawback of generating interpolated images is that the issues associated with the tumor motion hysteresis effects during breathing, that have been shown to exist in some patients, ${ }^{30}$ are completely ignored. The hysteresis effect pertains to the tumor, not the entire thorax, which prevented us from modeling image data for such scenarios, although local effects of this nature could certainly exist within the lung. Despite that, for Patient D, where hysteresis was present, the analysis of the cumulative doses using both the actual and the simulated data suggests that assuming a linear trajectory between exhale and inhale-essentially splitting the difference between the exhale to inhale path and the inhale to exhale return path-could generate sufficiently accurate predictions for clinically relevant metrics derived from the cumulative doses.

While the reason for why cumulative doses evaluated in various scenarios are similar is not immediately obvious, we believe that the effects are a consequence of the fact that the dose received by each anatomical unit as it progresses through each breathing phase changes in time in a somewhat linear fashion. Assuming that a patient's respiratory pattern is such that a breathing function

$$
f=F(t)
$$

exists, where $f=\frac{z_{0}-z(t)}{b}$ is the fractional distance away from the $z_{0}$ exhale coordinate, $b$ the breathing amplitude, and $z(t)$ the excursion at time $t$, then, the breathing probability distribution function $p(f)$ can be defined by

$$
p(f) d f=\frac{d t}{\tau},
$$

\begin{tabular}{|c|c|c|c|c|c|c|c|c|}
\hline \multirow[b]{2}{*}{ Fit } & \multirow[b]{2}{*}{ Method } & \multicolumn{3}{|c|}{ Patient A } & \multicolumn{2}{|c|}{ Patient B } & \multicolumn{2}{|c|}{ Patient C } \\
\hline & & $\operatorname{MLD}(G y)$ & $\operatorname{gEUD}(\mathrm{Gy})$ & $\begin{array}{l}\text { Esophagus } \\
\text { NTCP(\%) }\end{array}$ & $\operatorname{MLD}(\mathrm{Gy})$ & gEUD(Gy) & $\operatorname{MLD}(G y)$ & gEUD(Gy) \\
\hline \multirow[t]{5}{*}{$n=1$} & "20 state" & 4.33 & 109.7 & 6.07 & 12.62 & 95.8 & 7.76 & 69.1 \\
\hline & "10 state" & 4.32 & 109.5 & 5.87 & 12.60 & 95.6 & 7.75 & 69.0 \\
\hline & "2 state" & 4.33 & 109.4 & 5.78 & 12.60 & 95.5 & 7.72 & 69.1 \\
\hline & "2-ave state" & 4.32 & 109.7 & 6.06 & 12.61 & 95.6 & 7.76 & 68.9 \\
\hline & CT25(ave) & 4.31 & 109.3 & 5.94 & 12.57 & 95.5 & 7.75 & 68.9 \\
\hline \multirow[t]{5}{*}{$n=3$} & "20 state" & 4.06 & 109.5 & 5.48 & 12.74 & 96.2 & 7.57 & 69.1 \\
\hline & "10 state" & 4.06 & 109.4 & 5.42 & 12.73 & 96.1 & 7.56 & 69.0 \\
\hline & "2 state" & 4.05 & 109.2 & 5.39 & 12.71 & 95.9 & 7.54 & 69.1 \\
\hline & "2 ave state" & 4.05 & 109.4 & 5.13 & 12.74 & 96.2 & 7.56 & 68.8 \\
\hline & CT35(ave) & 4.00 & 109.6 & 5.14 & 12.74 & 96.3 & 7.58 & 69.1 \\
\hline
\end{tabular}

TABLE IV. Treatment metrics for all scenarios used to estimate the cumulative dose, for $n=1$ and $n=3$. 


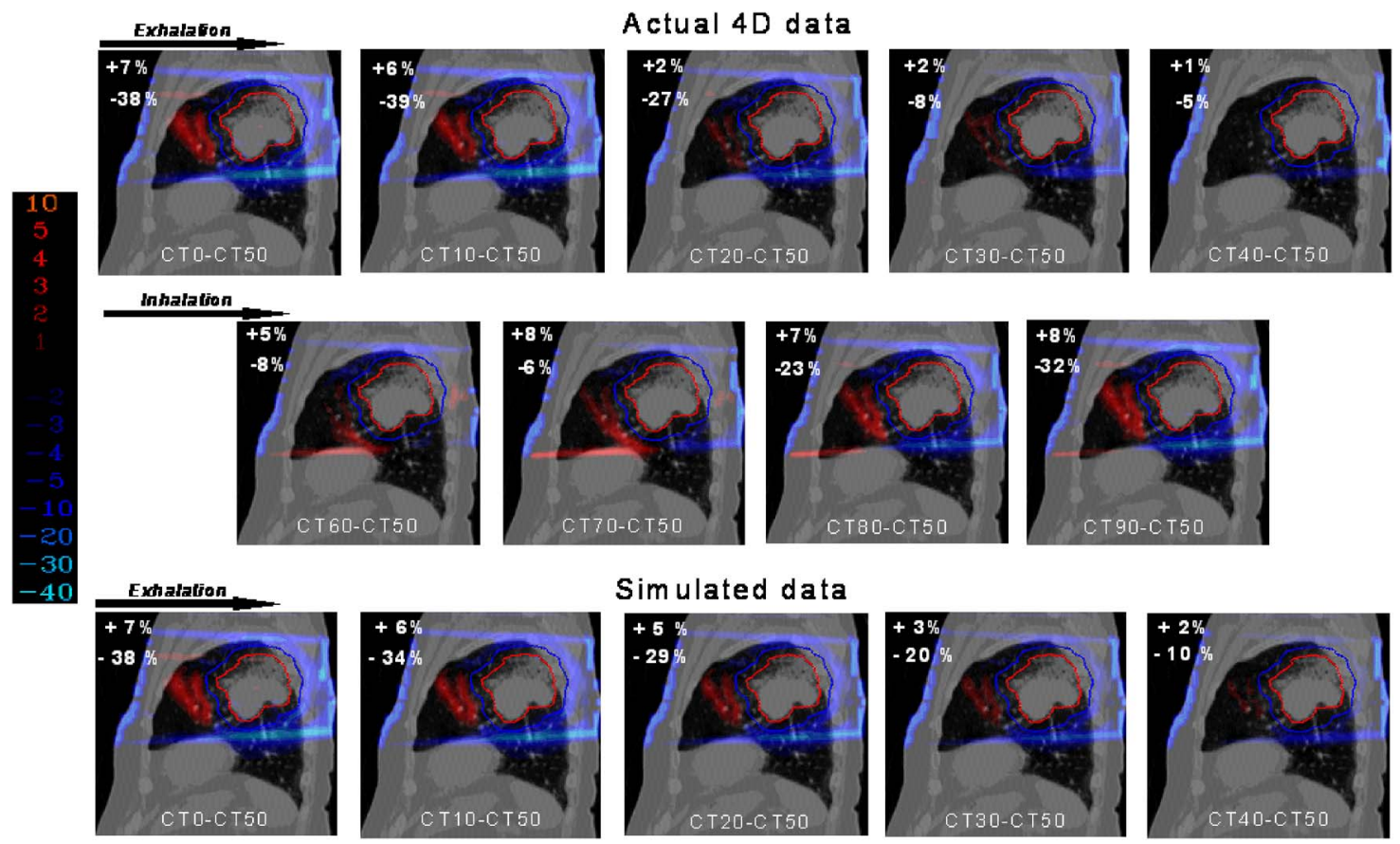

FIG. 7. Patient D: dose differences between doses in each actual or simulated dataset and the exhale planning dataset. The numbers indicate maximum/ minimum point dose differences inside the lung. Red/blue spots indicate positive/negative differences.

meaning that the probability that a point lies within $d f$ around $f$ away from exhale is proportional to the amount of time $d t$ spent around that position relative to the breathing period $\tau$ (half period for no hysteresis). If the dose received by an anatomical voxel at each breathing phase is proportional to the fraction away from exhale,

$$
D_{f}=f \cdot\left(D_{E}-D_{I}\right)+D_{E}=k f+k_{0},
$$

then the cumulative dose at a given "cell" (in our case exhale voxel) over the breathing cycle, when an infinite number of intermediate states are considered $\left(D_{\infty}\right)$, equals the dose received at the time-averaged phase of the breathing cycle $\left(D_{f_{\text {ave }}}\right)$. This is independent of the specific form of the breathing pattern (if reproducible); however, an explicit breathing function is needed to determine the average position during the breathing cycle.

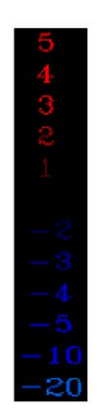

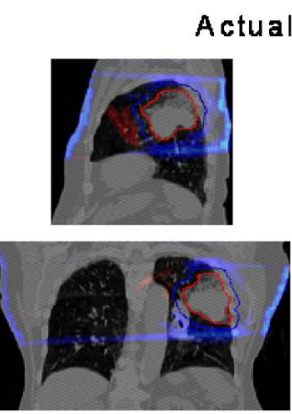

"10-state" - Exhale
Actual 4D data
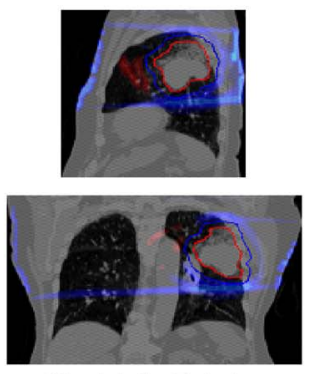

"2-state" - Exhale
The assumption of a linear change in dose distribution shape between breathing states technically would hold true if a "point" moved in a "dose cloud" of transient charged particle equilibrium (TCPE) within the dose distribution of a homogeneous media. However, this is never the case: the patient's anatomy is heterogeneous and, aside from the lack of TCPE in the buildup region of the depth dose curve, the linearity of the dose in space is violated in the vicinity of any interface between different media. Despite this, the cases analyzed in the current study suggest that although deviations from nonlinearity exist, the changes in doses received by voxels undergoing motion could in many cases be fit fairly well with linear dose change assumptions computed on a limited number of intermediate states. For the example patients investigated, the cumulative doses predicted by accumulating the doses from the "10-state," "2-ave state" and

FIG. 8. Patient D: dose differences between cumulative doses and the static (exhale) dose, estimated using the actual and the simulated datasets. The "10-state" and the "2-state" scenarios were used for the cumulative dose evaluation. The numbers indicate maximum/minimum point dose differences inside the lung. Red/blue spots indicate positive/negative differences. 

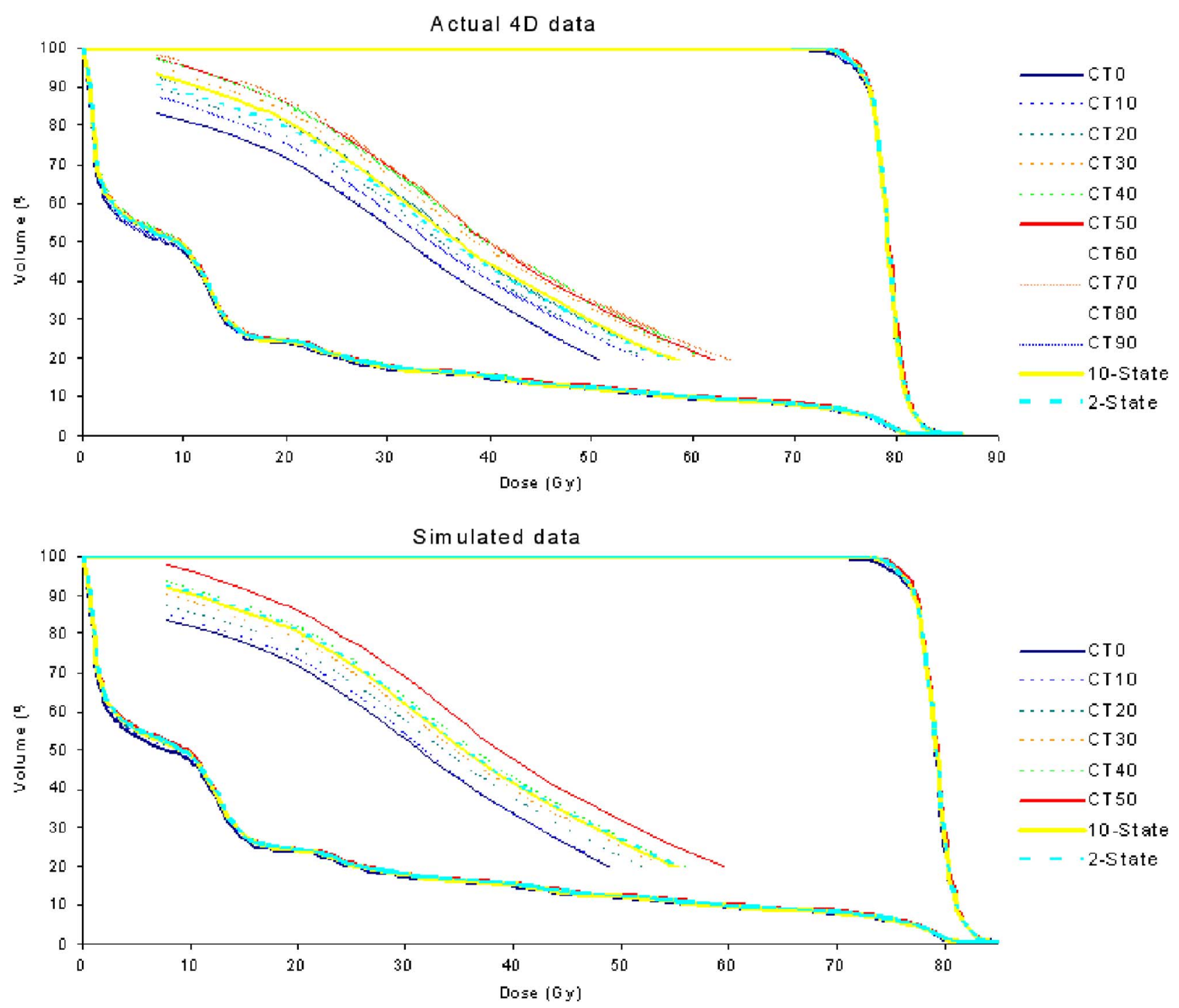

FIG. 9. Patient D: cumulative DVHs for tumor and normal lung tissue, for doses calculated using the actual and the simulated data, with insets showing details.

even the "2-state" models are in very good agreement with the more precise "20-state" model results. It should be noted though that the "20-state" model is in reality an over sampling of the data since the dose grid size used in the study was $3.5 \mathrm{~mm}$, while the maximum breathing amplitude observed for these patients was $2 \mathrm{~cm}$, translating into a $2.0 \mathrm{~mm}$ step size per $10 \%$ displacement away from exhale breathing phase. The agreements with the "20-state" results are also quite good when only the average states over the breathing function are used to predict the cumulative doses.

The actual impact of any nonlinearities in dose change in patients may be smaller than first thought for a variety of reasons. At interfaces, densities do not always change as abruptly as they might in phantom-designed experiments; for example, at the tumor margin the cell density most likely decreases gradually. Also, the use of multiple beams may lead to competing effects at a given point. Beyond these considerations, for parallel organs, especially of large volume such as lungs, any severe perturbations of the dose from a linear behavior likely occur in small regions and thus the effect of these perturbations on the total organ dose response may be too small to produce clinically significant changes. The variation in the esophagus NTCPs among the various scenarios used to estimate the cumulative dose appear to be somewhat larger than what was seen for lung. This is because the esophagus is a serial organ whose response to radiation is more sensitive to changes in the maximum dose-in other words, small changes in higher dose regions can produce larger NTCP variations for serial organs. Such changes do occur in the case of Patient A due to the fact that the esophagus was in the vicinity of the beam edge. However, even in this case, the prescription dose would change less than 0.5 Gy in an iso-NTCP protocol if the "ave-state" esophagus NTCP were adjusted to match the "20-state" esophagus NTCP.

The current study investigated the problem of the number of breathing phases that need to be considered for accurate clinically relevant cumulative dose estimation for conformal treatment plans and $6 \mathrm{MV}$ photon beams. For higher beam energies, motion effects are not significantly larger, as shown 


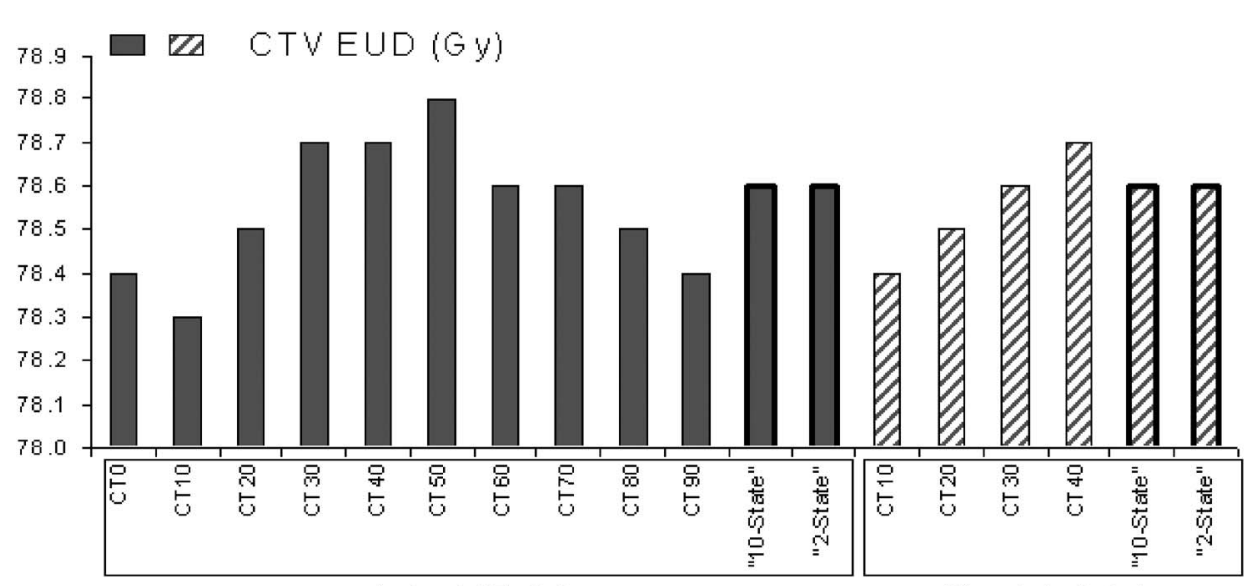

Actual 4D data

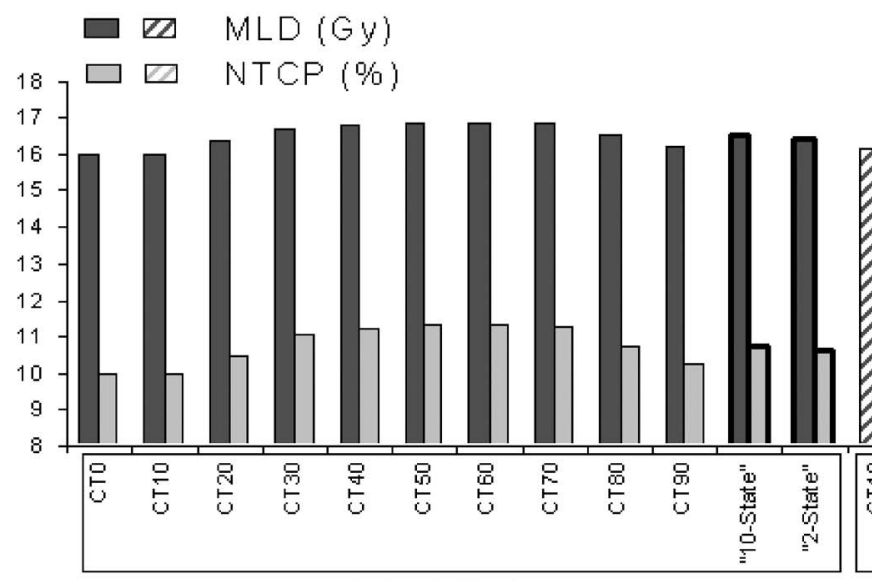

Actual 4 D data
Simulated data

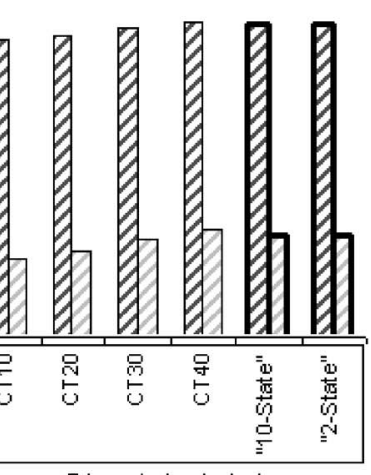

Sim ulated data
FIG. 10. Patient D: CTV EUD(Gy), MLD(Gy) and lung NTCP(\%) calculated for all intermediate states (from the actual and simulated datasets) and for cumulative doses. in a recent study, ${ }^{31}$ and therefore it is highly unlikely that the accuracy of estimating cumulative dose by using fewer breathing phases would be significantly impacted by the use of more energetic beams. The topic has been recently addressed for intensity modulated radiation therapy (IMRT) as well. Flampouri et $a l .{ }^{32}$ have found that when using threephase cumulative doses, less than $2.5 \%$ of the CTV sees doses that differ with more than $3 \%$ from doses calculated using ten phases.

As already mentioned, the average phase of the respiratory cycle can describe fairly well the cumulative dose received during a full cycle (if the scored dose changes in a somewhat linear fashion) regardless of the specific shape of the breathing time-position probability pattern. However, a breathing pattern is needed in order to determine either what the average state actually is, or what the appropriate time weighting coefficients would be if exhale and inhale are to be used for the estimation of the cumulative dose. Therefore the reproducibility of the breathing pattern over the course of the treatment is important, though it is not yet well established how much reproducibility is good enough from a clinical standpoint. Engelsman et al. ${ }^{33}$ have shown, in a phantom study, that it is the systematic setup error rather than the random component of the setup uncertainty or the ventilatory motion that appears to have a dominant effect on the cumulative dose to targets. Lujan, Balter, and Ten Haken $^{34}$ suggested that a large deviation in the motion am- plitude between planning and delivery is a cause of concern, whereas in cases when the motion excursion varies around some nominal value it is unlikely that such variations would lead to clinically significant changes in the prescription doses. The same study ${ }^{34}$ pointed out that changes in the degree of asymmetry are likely to be significant only in worse case scenarios-for example, when the patient would not breathe freely during the delivery of the treatment, but would instead hold the breath at a respiratory phase far away from the one for which the treatment was designed. This finding is supported by the case of Patient D presented in this paper. As described in the previous section (Table II), the "2-state" scenario for the simulated data assumed time weights of 0.7 and 0.3 , respectively, for exhale and inhale, as calculated from the assumed breathing probability distribution function for $n=3$, whereas for the real data equal weights were used for exhale and inhale, a situation that technically would describe a symmetrical breathing cycle $(n=1)$. Despite that, the dose difference displays, the DVHs and the treatment metrics from Figs. 8-10 indicate similar results for essentially two different breathing asymmetries, reinforcing conclusions from prior work that moderate variations in the breathing pattern are unlikely to impact significantly the metrics customarily used to assess treatment plans.

To date there is evidence ${ }^{30,35,36}$ that important changes can occur in the mean tumor position during treatment. This, however, does not invalidate our findings. Instead, if a care- 
ful monitoring of the respiration indicates a change in the average tumor position that is believed to be clinically important, the cumulative dose to date and the predicted total dose for the full treatment can be reevaluated based on either the dose received at the new average respiratory phase or by adjusting correspondingly the weighting coefficients for the exhale and inhale geometries.

It should also be noted that designing a treatment at the breathing phase that corresponds to the mean target position-in cases where it is possible to obtain that timeweighted average state, for example, from a 4D datasetappears to offer the advantage of minimizing the size of the CTV-PTV expansion, ${ }^{37-40}$ which is the ultimate goal of any attempt to deal with breathing induced motion. When it is not feasible to obtain the average state, use of only exhale and inhale scans from a patient could suffice, and this may be a more simply implemented data gathering method. This would still require the ability to perform distorted image registration between the inhale and exhale datasets and knowledge of an appropriate breathing function, both to be used to either simulate and then plan with and compute dose on an average breathing state, or use weighting factors to score dose on (e.g.) the exhale dataset if the patient is to be treated while breathing freely.

\section{ACKNOWLEDGMENTS}

This work was supported by NIH Grant Nos. P01 CA59872 and R01 CA106770. The authors would like to thank Dan Tatro, C.M.D., for the help provided with treatment planning and Lujun Zhao, M.D., for helping with structure segmentation.

${ }^{\text {a) }}$ Presented in part at the 47th Annual Meeting of the American Association of Physicists in Medicine, July 24-28, 2005, Seattle, Washington.

${ }^{b)}$ Electronic-mail: mrosu@med.umich.edu

${ }^{c}$ Present address: University of Nebraska Medical Center, Department of Radiation Oncology, Omaha, NE 68198-7521.

${ }^{1}$ J. M. Balter et al., "Uncertainties in CT-based radiation therapy treatment planning associated with patient breathing," Int. J. Radiat. Oncol., Biol., Phys. 36, 167-174 (1996).

${ }^{2} \mathrm{~S}$. Shimizu et al., "Impact of respiratory movement on the computed tomographic images of small lung tumors in three-dimensional (3D) radiotherapy,” Int. J. Radiat. Oncol., Biol., Phys. 46, 1127-1133 (2000).

${ }^{3}$ J. Wong et al., "The use of active breathing control (ABC) to reduce margin for breathing motion," Int. J. Radiat. Oncol., Biol., Phys. 44, 911-999 (1999).

${ }^{4}$ L. A. Dawson et al., "The reproducibility of organ position using active breathing control $(\mathrm{ABC})$ during liver radiotherapy," Int. J. Radiat. Oncol., Biol., Phys. 51, 1410-1421 (2001).

${ }^{5}$ J. Hanley et al., "Deep inspiration breath-hold technique for lung tumors: The potential value of target immobilization and reduced lung density in dose escalation," Int. J. Radiat. Oncol., Biol., Phys. 45, 603-611 (1999). ${ }^{6} \mathrm{~K}$. E. Rosenzweig et al., "The deep inspiration breath-hold technique in the treatment of inoperable non-small-cell lung cancer," Int. J. Radiat. Oncol., Biol., Phys., 48, 81-87 (2000).

${ }^{7} \mathrm{D}$. Mah et al., "Technical aspects of the deep inspiration breath-hold technique in the treatment of thoracic cancer," Int. J. Radiat. Oncol., Biol., Phys. 48, 1175-1185 (2000).

${ }^{8}$ S. S. Vedam et al., "Acquiring a four-dimensional computed tomography dataset using an external respiratory signal," Phys. Med. Biol. 48, 45-62 (2003).

${ }^{9} \mathrm{D}$. Low et al., A method for the reconstruction of four-dimensional synchronized CT scans acquired during free breathing, Med. Phys. 30, 1254-1263 (2003).
${ }^{10} \mathrm{~T}$. Pan et al., "4D-CT imaging of a volume influenced by respiratory motion on multi-slice CT," Med. Phys. 31, 333-340 (2004).

${ }^{11}$ P. J. Keall et al., "Time-The fourth dimension in radiotherapy (ASTRO Panel Discussion)," Int. J. Radiat. Oncol., Biol., Phys., 57, S8-9 (2003).

${ }^{12}$ G. C. Sharp et al., "Prediction of respiratory tumour motion for real-time image-guided radiotherapy," Phys. Med. Biol. 49, 425-440 (2004).

${ }^{13}$ S. S. Vedam et al., "Predicting respiratory motion for four-dimensional radiotherapy," Med. Phys. 31, 2274-2283 (2004).

${ }^{14}$ A. E. Lujan et al., "A method for incorporating organ motion due to breathing into 3D dose calculations," Med. Phys. 26, 715-720 (1999).

${ }^{15}$ M. Unser, "Splines: a perfect fit for signal and image processing," IEEE Signal Process. Mag. 16, 22-38 (1999).

${ }^{16}$ M. L. Kessler and M. Roberson, "Image registration and data fusion for radiotherapy treatment planning," in New Technologies in Radiation, edited by W. Schlegel, T. Bortfeld, and A. L. Grosu (Springer-Verlag, New York, 2005), pp. 41-52.

${ }^{17}$ M. L. Kessler et al., "Deformable image registration using multiresolution B-splines," Med. Phys. 31, 1792 (2004).

${ }^{18}$ J. Nocedal and S. Wright, Numerical Optimization (Springer-Verlag, New York, 1999).

${ }^{19}$ M. J. Fitzpatrick et al., "Displacement-based binning of time-dependent computed tomography image data sets," Med. Phys. 33, 235-246 (2006).

${ }^{20} \mathrm{~N}$. Wink, C. Panknin, and T. D. Solberg, "Phase versus amplitude sorting of 4D-CT data," J. Appl. Clin. Med. Phys. 7, 77-85 (2006).

${ }^{21}$ E. Schreibmann, G. T. Chen, and L. Xing, "Image interpolation in 4D CT using a B-Spline deformable registration model," Int. J. Radiat. Oncol., Biol., Phys. 64, 1537-1550 (2006).

${ }^{22}$ J. Sempau, S. J. Wilderman, and A. F. Bielajew, "DPM, a fast, accurate Monte Carlo code optimized for photon and electron radiotherapy treatment planning dose calculations," Phys. Med. Biol. 45, 2263-2291 (2000).

${ }^{23}$ I. J. Chetty et al., "Photon beam relative dose validation of the DPM Monte Carlo code in lung-equivalent media," Med. Phys. 30, 563-573 (2003).

${ }^{24}$ I. J. Chetty et al., "Reporting and analyzing statistical uncertainties in Monte Carlo-based treatment planning," Int. J. Radiat. Oncol., Biol., Phys. 65, 1249-1259 (2006).

${ }^{25} \mathrm{M}$. Rosu et al., "Dose reconstruction in deforming lung anatomy: Dose grid size effects and clinical implications," Med. Phys. 32, 2487-2495 (2005).

${ }^{26} \mathrm{~A}$. Niemierko, "A generalized concept of equivalent uniform dose (EUD)," Med. Phys. 26, 1100 (1999).

${ }^{27}$ J. Lyman, "Complication probability as assessed from dose volume histograms," Radiat. Res. 104, S13-19 (1985).

${ }^{28}$ G. J. Kutcher and C. Burman, "Calculation of complication probability factors for non-uniform normal tissue irradiation: The effective volume method,” Int. J. Radiat. Oncol., Biol., Phys. 16, 1623-1630 (1989).

${ }^{29}$ Y. Seppenwoolde et al., "Comparing different NTCP models that predict the incidence of radiation pneumonitis. Normal tissue complication probability,” Int. J. Radiat. Oncol., Biol., Phys. 55, 724-735 (2003).

${ }^{30}$ Y. Seppenwoolde et al., "Precise and real-time measurement of 3D tumor motion in lung due to breathing and heartbeat, measured during radiotherapy," Int. J. Radiat. Oncol., Biol., Phys. 53, 822-834 (2002).

${ }^{31} \mathrm{M}$. Rosu et al., "The impact of 4D breathing motion effects versus tissue heterogeneity in lung cancer treatment planning," Med. Phys. 33, 2232 (2006).

${ }^{32}$ S. Flampouri et al., "Estimation of the delivered patient dose in lung IMRT treatment based on deformable registration of 4D-CT data and Monte Carlo simulations," Phys. Med. Biol. 51, 2763-2779 (2006).

${ }^{33} \mathrm{M}$. Engelsman et al., "The effect of breathing and set-up errors on the cumulative dose to a lung tumor," Radiother. Oncol. 60, 95-105 (2001).

${ }^{34}$ A. E. Lujan, J. M. Balter, and R. K. Ten Haken, "A method for incorporating organ motion due to breathing into 3D dose calculations: Sensitivity to variations in motion," Med. Phys. 30, 2643-2649 (2003).

${ }^{35} \mathrm{~L}$. Kestin et al., "Breathing variation during thoracic radiations for adaptive radiotherapy of lung cancer," Int. J. Radiat. Oncol., Biol., Phys. 60, S610 (2004).

${ }^{36} \mathrm{M}$. J. Murphy et al., "The effectiveness of breath-holding to stabilize lung and pancreas tumors during radiosurgery," Int. J. Radiat. Oncol., Biol., Phys. 53, 475-482 (2002).

${ }^{37} \mathrm{D}$. Yan et al., "Target margin for respiratory motion: Optimal patient/ organ positioning for treatment planning is critical," Radiother. Oncol. 64, Supplement 1, S259-S260 (2002). 
${ }^{38}$ J. Liang et al., "Minimization of target margin by adapting treatment planning to target respiratory motion," Int. J. Radiat. Oncol., Biol., Phys., Suppl. 57, Suppl. 1, S233-S234 (2003).

${ }^{39}$ J. W. Wolthaus et al., "Mid-ventilation CT scan construction from fourdimensional respiration-correlated CT scans for radiotherapy planning of lung cancer patients,” Int. J. Radiat. Oncol., Biol., Phys. 65, 1560-1571 (2006).

${ }^{40}$ M. Schwarz et al., "Impact of geometrical uncertainties on 3D CRT and IMRT dose distributions for lung cancer treatment," Int. J. Radiat. Oncol., Biol., Phys. 65, 1260-1269 (2006). 